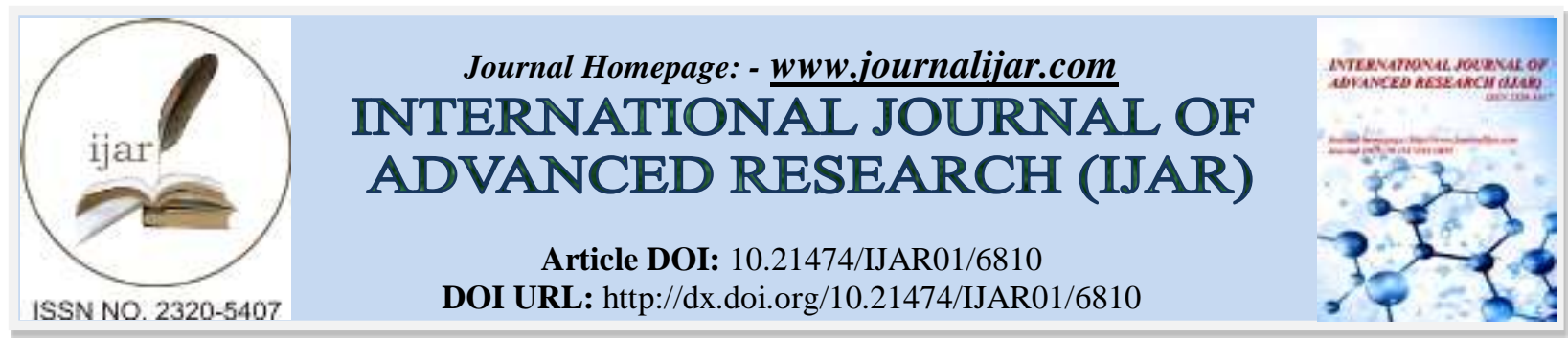

RESEARCH ARTICLE

\title{
IMPACT OF SHOCK WAVE THERAPY ON FROZEN SHOULDER PATIENTS - PAIN AND FUNCTIONS.
}

\footnotetext{
"Ahmed Abdelmoniem Ibrahim. Ph D. PT. ${ }^{1}$, Mohamed raffat Mohamed atteya. Ph. D. PT ${ }^{2}$, Ehab Mohamed Kamel: Ph. D. PT ${ }^{2}$, Ibrahim Jazza Alanzi ${ }^{2}$, Ali Rbein $\mathrm{Ali}^{2}$ and Abdullah Salim Hamad Albuwait ${ }^{2}$.

1. physiotherapy Department, Cairo university hospitals, Cairo; Egypt pox -11562 .

2. Physical Therapy Department, College of Applied medical sciences, University of Hail-KSA -pox.2440.
}

\section{Manuscript Info}

Manuscript History

Received: 22 January 2018

Final Accepted: 24 February 2018

Published: March 2018

Keywords:-

shock wave therapy, Pain, Function.

\section{Abstract}

Purpose: The present study was conducted to examine the effects of shock wave therapy on frozen shoulder patients' pain and functions. Subjects and Methods: 30 frozen shoulder patients were divided into two groups, 15 patients for each group, group I (GI) received shock wave therapy in addition to a program of exercise therapy and group (GII) received only exercise program .Visual analog scales were used to measure frozen shoulder patients' pain, and patient-specific functional scales were used to evaluate the degree of functional disorders. Results: the two groups showed significant decreases in terms of visual analog scales and patient-specific functional scales, although the group I (GI) showed significantly lower scores than the group (GII). Conclusion: shock wave therapy is considered an effective intervention for improving frozen shoulder patients' pain and functions.

\section{Introduction:-}

Adhesive capsulitis (frozen shoulder) occurs in approximately 2-5\% of the general population which is 2-4 times more common in women than men, and is most frequently seen in individuals between 40 and 60 years of age the disease is more common in patients with diabetes mellitus, rotator cuff lesions, thyroid disorders, Chronic Obstructive Pulmonary Diseases, cerebrovascular accident, myocardial (Lin et al, 2006).

FS results in a gradual loss of shoulder range of motion (ROM) and strength of surrounding muscles. The increase of the shoulder active ROM and strength of the shoulder muscles and decrease of the pain are important component for reduction of physical disability and improvement of the shoulder function in of FS patients during rehabilitation (Frontra, 2008).

The shoulder adhesive capsulitis (frozen shoulder) is a common illness, which causes significant morbidity. It usually occurs in the sixth decade of life with age peak 56 years, and the condition occurs slightly frequent in women than in men (Uppal et al, 2015).

The term frozen shoulder was first described by Duplay in 1872, however only in 1934 it was first used by Codmam. Such pathology was defined by Codmam as being of a slow onset, with painful symptomatology close to deltoid muscle insertion, inability to sleep on the affected side, and restriction in both active and passive elevation 
and external rotation, but with a normal radiological appearance, definition also attributed the consensus of orthopedic surgeons from the American Shoulder and Elbow surgeons (Manske et al, 2008).

In the absence of other pathological processes, a frozen shoulder diagnosis occurs with an insidious onset, night pain occurs, painful restraint of passive scapulohumeral elevation causing abduction of the shoulder less than 100 degrees, and external rotation of the shoulder less than half of normal (Braddom, 2011).

The history of the disease occurs in 3 phases: (1) freezing phase, characterized by diffuse pain and loss of movement (2-9 months); (2) the frozen phase, where the pain diminishes and the stiffness increases (4-12 months); and (3) thawing phase, characterized by a progressive normalization of mobility and function (4-12 months). The stages may last 1-3 years (Dalton, 2003).

The goals of treatment for patients with shoulder stiffness is to restore, maintain the range of motion (ROM) as well as the functionality, and reduce the painful symptoms. Treatments for shoulder adhesive capsulitis include rehabilitation as the first conservative measure, anti-inflammatories, intra-articular corticosteroids, capsular distension injections, and surgical interventions in refractory cases. In the context of the therapy it is possible to highlight the technical mobilization and manipulation for restoring a pain-free state and normal use of the upper limb (Ide and Takagi, 2004).

Although frozen shoulder is considered self-limiting, long term longitudinal follow-up data are scarce. The largest longitudinal series to date (223 patients) showed that $41 \%$ of patients had persistent symptoms at a mean follow up of 4.4 years, but this was based in secondary care, where more severe and less tractable cases are likely to be seen (Hand et al, 2007). The same study found recurrence rare, but the other shoulder was affected in 6-17\% of patients within five years. Reported associations include diabetes, cardiovascular disease, trauma, stroke, neurosurgery, and thyroid disease (Maund et al, 2012).

Manual therapy consists of a series of operations, including manual techniques (muscle mobilization, specific joint mobilization, coordination or stabilization), such interventions are widely used by chiropractors, in the osteopathy or by physiotherapists (Bos et al, 2003).

Physical therapy modalities and exercises can be listed as the most common treatment for shoulder pain (Arsian and Celiker, 2001). Exercises and muscle stretching are effective means of treating shoulder dysfunction and enhancing range of motion in patients with shoulder impingement (Conory and Hayes, 1998).

Shock waves are sound waves that are generated by a source that creates vibration which are then transported through tissue via fluid and solid particles interaction (Perez et al., 2003). It has been used for the treatment of numerous musculoskeletal disorders, including calcified tendonitis of shoulder, lateral epicondylitis, achilles and patellar tendinopathies, chronic planter fasciitis, osteonecrosis of the femoral head, and delayed union and non-union of fractures (Furia, 2006)

In the last ten years, shock wave therapy has been successfully employed as an anti-inflammatory therapy in a number of orthopedic diseases such as pseudoarthrosis, tendinitis calcarea of the shoulder epicondylitis, plantar fasciitis, and several inflammatory tendon diseases (Ciampa et al, 2005).

Physical therapy modalities are often the first line of management for shoulder pain.

Conservative treatment of patients with impingement symptoms commonly includes therapeutic exercise programs intend to restore normal kinematics and muscular control of scapula (Lynch, 2002).

It seems reasonable to use treatment modalities aimed at rapid recovery rate with a minimum number of sessions, this would minimize the negative effects of a Hospital-based rehabilitation procedure on patient's daily life and their subsequent compliance (Bang and Deyle, 2000). A faster program with fewer treatment sessions not only enables the patients to proceed with most of their daily activities but also decrease the cost of treatment, this would reflect an additional health care advantage for the treatment of impingement syndrome (Guler-Uysal and Kozanoglu, 2004). 


\section{Methodology:-}

The study was conducted in King Khalid Hospital, Hail, KSA to determine the effect of extracorporeal shock wave therapy on frozen shoulder patients' pain and functions.

\section{Subjects:-}

Thirty subjects recruited from King Khalid Hospital, with a mean age of $56.1 \pm 10.6$ years, mean body height of $174.9 \pm 6.0 \mathrm{~cm}$, mean body weight of $64.7 \pm 6.9 \mathrm{~kg}$, and mean body mass index of $22.5 \pm 2.8$. All subjects signed an informed consent form prior to participation. The study protocol was approved by the University's ethics committee.

\section{Inclusion criteria:-}

Participants with FS, age from 40 to 70 and body mass index not more than 30 .

\section{Exclusion criteria:-}

Exclusion criteria were

1. history of previous surgery on the shoulder,

2. history of shoulder fracture, cancer, inflammatory disorders, bleeding disorders,

3. unwillingness to participate in the study.

\section{Group assignment:-}

The subjects were randomly assigned into two groups of equal numbers each containing twenty patients group I (GI) $(\mathrm{N}=15)$ received extracorporeal shock wave therapy in addition to a program of exercise therapy and group $(\mathrm{GII})(\mathrm{N}=15)$ received only the same program of exercise given to GI.

\section{Instrumentations for evaluation}

Evaluation were conducted for each patient of the two groups before and after treatment. The assessment procedure included the following:

- Pain assessment:

Pain were assessed by using visual analogue scale (VAS). (Grant et al, 1999).

- functional disability:

Disability has been assessed by using PSFS: Patient-specific functional scale. This useful questionnaire were used to quantify activity limitation and measure functional outcome for patients with any orthopaedic condition

- Extracorpreal shockwave device:

It is a device that provide shock wave its model is radial spec and serial number is (1107394) medispec. It is connected to electrical main supply 115/220VA C at frequency 50/60 HZ and $5 \mathrm{~A}$.

- The exercise program was applied as follows:

- Passive stretching exercise for the posterior shoulder capsule and surrounding musculature .

- Strengthening exercises for shoulder flexion, rowing, and horizontal extension.

Each exercise was performed as 3 sets of 10 repetitions with a 60-seconds rest period between, each set.

The shock wave therapy was administrated using $15 \mathrm{~mm}$ head applicator. Each patient in the experimental group was received 3 sessions one session per week for three consecutive weeks with 6000 impulses, an energy flex density of $0.22 \mathrm{~mJ} / \mathrm{mm} 2$, pulse rate $10 / \mathrm{sec}$ and frequency $1-15 \mathrm{~Hz}$. The treatment area prepared with a coupling gel to minimize the loss of shock wave energy at the interface between applicator tip and skin.

\section{Statistics analysis:-}

All data were presented as a mean and standard deviation. t-test was used to compare the differences between values. The accepted level of significance for differences was equal to or less than 0.05 for all tests $(\mathrm{P}$ value $<0.05)$.

\section{Results:-}

In comparisons of VAS and PSFS, both the group I and the group (GII) showed significant decreases in both VAS and PSFS In intergroup comparisons of VAS and PSFS, the group I showed significantly lower scores than the group (GII) (Table 2) and (figure 2). 
Table 1:- demographic data (age ,height, weight, body mass index)

\begin{tabular}{|l|l|}
\hline Parameter & Value \\
\hline age & 56.1 \\
\hline height & 174.9 \\
\hline weight & 64.7 \\
\hline body mass index & 22.5 \\
\hline
\end{tabular}

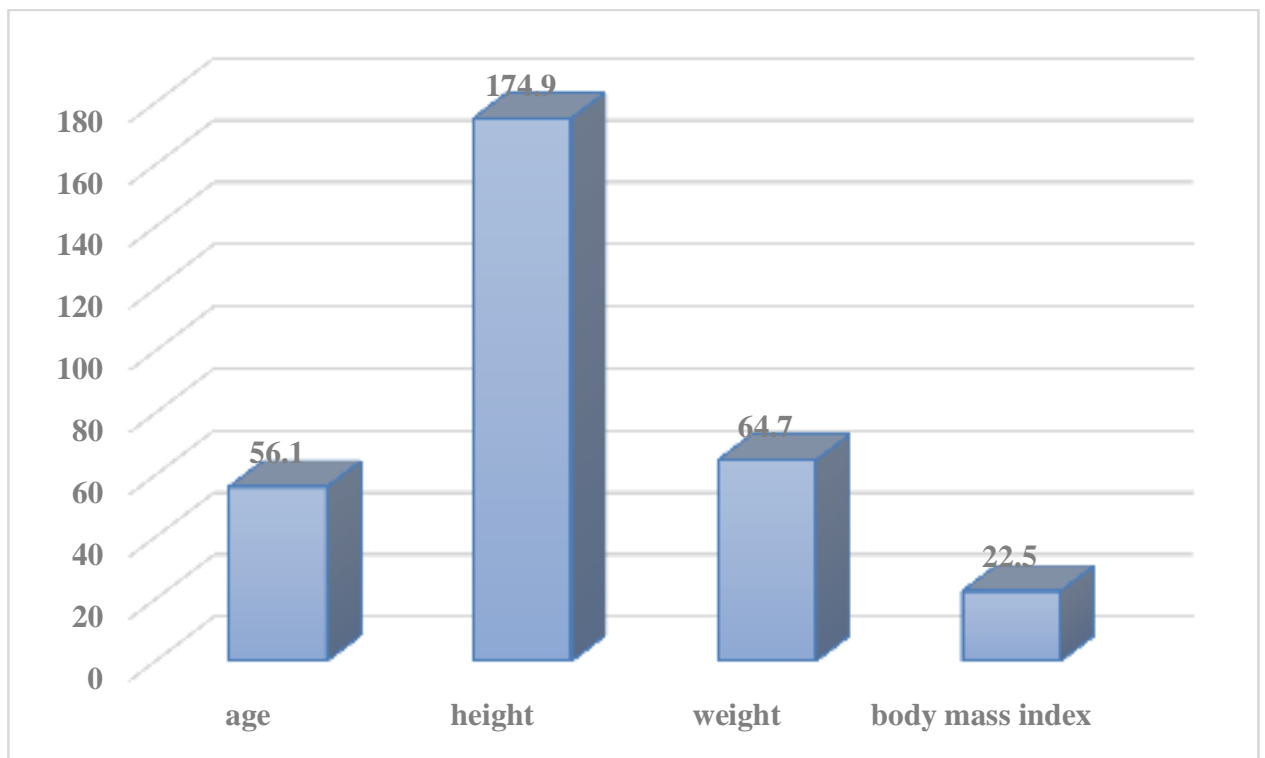

Fig 1:- demographic data (age ,height, weight, body mass index)

Table 2:- Comparison of VAS and PSFS for each group (VAS: visual analog scale, PSFS: Patient-specific functional scale,

\begin{tabular}{|l|l|l|l|}
\hline & Group & Pre & Post \\
\hline \multirow{2}{*}{ VAS (mm) } & group (GII) & $7.3 \pm 1.1$ & $5.7 \pm 0.9$ \\
\cline { 2 - 4 } & group I (GI) & $7.3 \pm 1.0$ & $3.9 \pm 1.2^{\dagger \dagger}$ \\
\hline PSFS (point) & group (GII) & $7.7 \pm 0.8$ & $6.1 \pm 1.2$ \\
\cline { 2 - 4 } & group I (GI) & $7.7 \pm 0.8$ & $3.8 \pm 0.9^{\dagger \dagger}$ \\
\hline
\end{tabular}




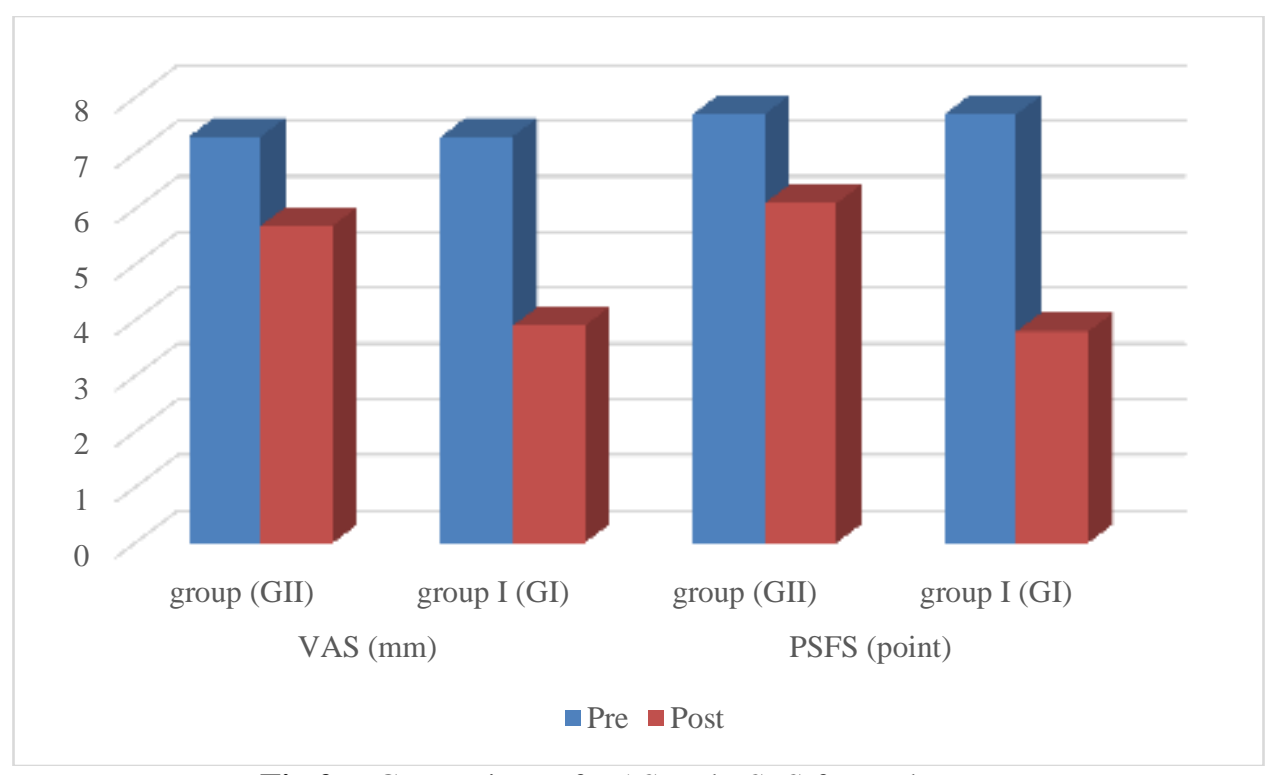

Discussion:-

Fig 2:- Comparison of VAS and PSFS for each group

Frozen shoulder (FS) is a painful condition that most commonly affects people in their 50s and may limit daily activities and disturb sleep. It is an idiopathic and progressive disease, which is identified by pain and decreased range of motion (ROM) of the shoulder and shoulder joint capsule fibrosis (Dawson et al, 2010).

The rational for the use of shock wave for these conditions is based on stimulation of soft tissue healing by local hyperemia, revascularization, reduction of calcifications, inhibition of pain and denervation to achieve pain relief and persistent healing of chronic inflammatory process. Side effects are usually minimal, and may include local bruising or short duration swelling and tenderness (Lebrun, 2005).

In the present study, in intra-group comparisons of VAS and PSFS, both groups showed significant decreases in both VAS and PSFS. In comparisons of VAS and PSFS, the group I significantly lower scores than the group (GII). Given these results, ESWT is considered to be effective for pain relief. It uses fine, repetitive stimuli produced by a sound wave that can be spread through soft tissues without energy dissipation. In doing this, it causes changes in cells' metabolism and the permeability of endothelial cell tissues, leading to pain relief, and has positive effects on soft tissues. (Loew M.,et al 1999) (Seidl ,m et al M1994)

The limitations of this study were as follows: the emphases were on the treatment effect because the patients who wanted to receive shock wave were assigned in the treatment group, on the improvement in symptoms due to natural death of calcific tendinitis, and on the effect of nonsteroidal anti-inflammatory drugs on the treatment outcome. However, this study showed that low-volume shock wave was also effective for calcific tendinitis. A better-controlled experimental group and standard protocol for maximizing the treatment effect of shock wave on calcific tendinitis will be needed in future studies. 


\section{References:-}

1. Arsian S and Celiker R (2001): Comparison of the efficacy of local corticosteroid and physical therapy for the treatment of adhensive capsality. Rheumatology Int; 21: 20-23.

2. Bang M.D. and Deyle G.D.: Comparison of supervised exercises with and without manual physical therapy for patients with shoulder impingement syndrome. J. Orthop. Phys. Ther., 30: 130-137, 2000.

3. Braddom RL. 4th ed. Philadelphia: WB Saunders;; 2011. Handbook of Physical Medicine and Rehabilitation;; p. 825.

4. Ciampa A.R., Prati A.C., Amelio E., Cavalieri E., Persichini T., et al,. Nitric oxide mediates anti-inflammatory action of extracorporeal shock waves. FEBS Letters, 579 (19): 6839-6845, 2005.

5. Codman EA. Tendinitis of the Short Rotators. In: The Shoulder: Rupture of the Supraspinatus Tendon and Other Lesions in or about the Subacromial Bursa. Boston MA: Thomas Todd, 1934.

6. Conory DE and Hayes KW (1998): The effect of joint mobilization as a component of comprehensive treatment for primary shoulder impingement syndrome. J. Orthop. Sports Phys. Ther; 28:3-14.

7. Dalton SE. The shoulder. In: Hochberg M, Silman AJ, Smolen JS, editors. Rheumatology. 3rd ed. Toronto: Mosby;; 2003. pp. 615-30.

8. Dawson J, Shepperd S, Carr A. An overview of factors relevant to undertaking research and reviews on the effectiveness of treatment for frozen shoulder. Shoulder Elbow 2010;2:232-7doi:10.1111/j.17585740.2010.00067.x.

9. Duplay E. De la periarthrite scapulo-humérale et des raideurs de l'épaule quien sont la conséquence. Arch Gen Med 1872;20:513-542.

10. Frontra G. Essentials of Physical Medicine and Rehabilitation. 2nd ed. Philadelphia: Saunders, Elsevier;; 2008. Adhesive capsulitis;; pp. 49-54.

11. Furia JP (2006): High energy extracorporeal shock wave therapy for insertional achillis tendinopathy. Am J sports Med; 34: 733-740.

12. Grant S., Aitchison T., Henderson E., Christie J., Zare S., et al. A comparison of the reproducibility and the sensitivity to change of visual analogue scales, borg scales, and likert scales in normal subjects during submaximal exercise, 10: 1165-1168, 1999.

13. Guler-Uysal F. and Kozanoglu E.: Comparison of the early response to two methods of rehabilitation in adhesive capsulitis. Swiss Med. Wkly, 134: 353-358, 2004.

14. Hand C, Clipsham K, Rees JL, Carr AJ. Long-term outcome of frozen shoulder. J Shoulder Elbow Surg 2008;17:231-6. doi:10.1016/j.jse.2007.05.009 pmid:17993282.

15. Ide J, Takagi K. Early and long-term results of arthroscopic treatment for shoulder stiffness. J Shoulder Elbow Surg 2004;13:174-9.

16. Korthals-de Bos IB, Müllner M, Hoving JL, Van Tulder MW, Rutten-van Mölken MP, Adèr HJ, et al. Cost effectiveness of physiotherapy, manual therapy, and general practitioner care for neck pain: economic evaluation alongside a randomised controlled trial Commentary: Bootstrapping simplifies appreciation of statistical inferences. Bmj. 2003;326(7395):911- 914.

17. Lebrun C (2005): Understanding shock wave therapy for the treatment of bone and tendon pain. Sportline; 1 (20): $1-4$.

18. Lin JJ, Lim HK, Yang JL. Effect of shoulder tightness on glenohumeral translation, scapular kinematics, and scapulohumeral rhythm in subjects with stiff shoulders. Journal of Orthopaedic Research 2006;24:1044-51.

19. Lynch S.A.: Surgical and non-surgical treatment of adhesive capsulitis. Curr. Opin. Orthop., 13: 15-27, 2002.

20. Loew M, Daecke W, Kusnierczak D, et al.: Shock-wave therapy is effective for chronic calcifying tendinitis of the shoulder. J Bone Joint Surg Br, 1999, 81: 863-867. [Medline] [CrossRef]

21. Manske RC, Prohaska D. Diagnosis and management of adhesive capsulitis. Curr Rev Musculoskelet Med 2008;1:180-9.

22. Maund E, Craig D, Suekarran S, et al. Management of frozen shoulder: a systematic review and costeffectiveness analysis. Health Technol Assess 2012;16:1-264. doi:10. 3310/hta16110. pmid:22405512.

23. Ombregt L. The shoulder. In: Ombregt L, Bisschop P, ter Veer HJ, eds. A system of orthopaedic medicine. Second ed. London: Churchill Livingstone, 2003:313-15.

24. Perez M, Weiner R and Gilley JC (2003): Extracorporeal shock wave therapy for the planter facilities. Clin. Pediatr. Med. Surg; 20: 333-346, 2003.

25. Uppal HS, Evans JP, Smith C. Frozen shoulder: a systematic review of therapeutic options. World J Orthop. 2015;6(2):263-8.

26. Seidl M, Steinbach P, Wörle K, et al.: Induction of stress fibres and intercellular gaps in human vascular endothelium by shock-waves. Ultrasonics, 1994, 32: 397-400. [Medline] [CrossRef] 